\title{
LOW INDEX MINIMAL HYPERSURFACES OF SPHERES*
}

\author{
OSCAR PERDOMO ${ }^{\dagger}$
}

\begin{abstract}
Let $M$ be a compact oriented non-equatorial minimal hypersurface of the unit ndimensional sphere. Suppose that for any non-zero vector in $w \in \mathbf{R}^{n+1}$ there exists an orthogonal matrix $B$ such that $B(M)=M$ and $B(w) \neq w$. Since all known examples of minimal hypersurfaces have antipodal symmetry, they satisfy this condition.

We prove that: i) the stability index of $M$ is greater than or equal to $n+2$ with strict inequality, unless $M$ is a Clifford hypersurface; ii) the difference between the first two eigenvalues of the Jacobi operator is less than or equal to $n-1$ with strict inequality, unless the norm of the second fundamental form is constant; and iii) if $M$ has antipodal symmetry and is not a Clifford hypersurface then the index is greater than $n+3$. Moreover if the unit normal vector is even, the index is greater than $2 n+2$.
\end{abstract}

1. Introduction. Let $M$ be a compact, oriented minimal hypersurface immersed in the $n$-dimensional sphere $S^{n}$. Let $\nu$ be a unit normal vector field along $M$. For any tangent vector $v \in T_{m} M, m \in M$, the shape operator $A$ is given by $A(v)=-\bar{\nabla}_{v} \nu$, where $\bar{\nabla}$ denotes the Levi Civita connection in $S^{n}$. We will denote by $\Delta$ the Laplacian on $M$. Given any function $f: M \longrightarrow \mathbf{R}^{1}$ we can form the 1-parameter variational family defined by

$$
M_{t}=\{\exp (m, t f(m) \nu): m \in M\}
$$

where $\exp (m, \cdot)$ is the exponential map at $m \in S^{n}$.

It is well known (see e.g. [9]) that the area of $M_{t}$ satisfies:

$$
\begin{gathered}
\left.\frac{d}{d t}\left(\operatorname{area}\left(M_{t}\right)\right)\right|_{t=0}=0 \quad(\text { minimality of } M) \\
\left.\frac{d^{2}}{d t^{2}}\left(\operatorname{area}\left(M_{t}\right)\right)\right|_{t=0}=\int_{M} J(f) f \quad \text { (second variation formula) }
\end{gathered}
$$

where $J$ is the Jacobi operator on $M$, given by

$$
J=-\Delta-\|A\|^{2}-(n-1)
$$

When $\int_{M} J(f) f<0$ for some $f \in C^{\infty}(M)$, we see that area $(M)>\operatorname{area}\left(M_{t}\right)$ for small values of $t \neq 0$. In particular this implies that the minimal hypersurface, $M$, while a critical point of the area functional, is not a local minimum. For hypersurfaces in spheres this is always the case, since we can take $f \equiv 1$.

The Jacobi operator induces a symmetric bilinear form

$$
I(f, g)=\int_{M} J(f) g
$$

known as the index form on $M$. Let $V$ be a subspace of $C^{\infty}(M)$ on which the index form is negative definite, i.e. $\int_{M} J(f) f<0$ for all $f \in V$. Since the eigenvalues

\footnotetext{
*Received February 17, 2001; accepted for publication March 6, 2001.

†Indiana University, Bloomington IN 47405, USA (osperdom@mafalda.univalle.edu.co).
} 
of the operator $-\Delta$ tend to $\infty$, the dimension of $V$ must be finite. We define the index of $M, \operatorname{ind}(M)$, as the maximum dimension of any such subspace $V$. Intuitively, $\operatorname{ind}(M)$ measures the number of "independent" directions in which $M$ fails to minimize area.

The easiest minimal hypersurfaces to describe are the equators, i.e. the totally geodesic $S^{n-1}$ 's in $S^{n}$, and the Clifford hypersurfaces, which are products of spheres; when we split the ambient space $\mathbf{R}^{n+1}$ as $\mathbf{R}^{k+1} \times \mathbf{R}^{l+1}$, with $k+l=n-1$ (and $k, l \geq 1$ ), we get a Clifford hypersurface corresponding to this splitting, namely

$$
S^{k}\left(\sqrt{\frac{k}{n-1}}\right) \times S^{l}\left(\sqrt{\frac{l}{n-1}}\right) \subset S^{n} \subset \mathbf{R}^{k+1} \times \mathbf{R}^{l+1}
$$

A minimal hypersurface $M$ with index $\operatorname{ind}(M)=0$ is called stable, i.e. it minimizes area up to second order. As seen above, in the sphere we always have $\operatorname{ind}(M) \geq 1$, so no minimal hypersurface is stable there. It is not hard to prove that totally geodesic hypersurfaces are characterized as the only minimal $M^{n-1} \subset S^{n}$ with $\operatorname{ind}(M)=1$, see for example Lemma 3.1.

Our efforts here begin with the simple observation that if $M$ is not totally geodesic, then $\operatorname{ind}(M)$ jumps; it is at least that of the Clifford hypersurfaces; namely, $n+2$ (see Lemma 3.1 again). This suggests the conjecture motivating our work:

Conjecture 1.1. The Clifford hypersurfaces are the only minimal $M^{n-1} \subset S^{n}$ with $\operatorname{ind}(M)=n+2$.

F. Urbano in [12], proved that the conjecture is true in the case $n=3$. The first part of our main theorem establishes that the conjecture is true in all dimensions, with one additional (and seemingly weak, as we will explain) assumption about the symmetries of $M$. That is, when ind $(M)=n+2$ and our weak symmetry assumption holds, $M$ is a Clifford hypersurface.

Conjecture 1.1 implies that the index functional jumps to at least $n+3$ for every non-equatorial minimal hypersurface which is not Clifford. Since for all known minimal hypersurfaces (different from equators and Clifford hypersurfaces) the index is a least $2 n+3$, we have the following conjecture:

Conjecture 1.2. ind $(M) \geq 2 n+3$ for any non-totally geodesic minimal hypersurface $M^{n-1} \subset S^{n}$ which is not Clifford.

In Section 4 we will partially solve this conjecture for minimal hypersurfaces with antipodal symmetry. We will show that it holds true for minimal hypersurfaces with antipodal symmetry and even unit normal vector $\nu$. For minimal hypersurfaces that define immersion in the projective space, those with odd unit normal vector field, Do Carmo-Ritore-Ros [3] showed the existence of an even eigenfunction of $J$ which is not a first eigenfunction. Combining this result with Theorem 1.3 part 1, we deduce that the index of any minimal hypersurface in $S^{n}$ with antipodal symmetry is greater than $\mathrm{n}+3$, provided that $M$ is not an equator or a Clifford hypersurface.

Let us motivate the second part of our main theorem. If $\|A\|$ is constant, then the first eigenvalue $\lambda_{1}$ of the Jacobi operator is $-(n-1)-\|A\|^{2}$. Because the coordinate functions are eigenvalues of the Laplacian with eigenvalue $-(n-1)$ we get that $-\|A\|^{2}$ is also an eigenvalue of $J$. Hence the second eigenvalue satisfies $\lambda_{2} \leq-\|A\|^{2}$, so we have that the distance between $\lambda_{1}$ and $\lambda_{2}$ is at most $n-1$, i.e.

$$
\lambda_{2}-\lambda_{1} \leq n-1
$$


The second part of our main theorem shows that, with the same assumption about symmetries as in part 1, the estimate above holds for minimal hypersurfaces even if $\|A\|$ is not constant. Moreover, equality in the estimate above implies $\|A\|^{2} \equiv\left|\lambda_{2}\right|$.

To state our assumption about the symmetries of $M$ we define the group

$$
O_{M}(n+1)=\{\gamma \in O(n+1): \gamma(M)=M\}
$$

The antipodal map, $\tau(x)=-x$, may or may not be an element of $O_{M}(n+1)$. When it is, we say that " $M$ has antipodal symmetry".

We shall prove:

THEOREM 1.3. Let $M$ be a compact, oriented minimal non-equatorial hypersurface of $S^{n}$, and suppose that $O_{M}(n+1)$ fixes only the origin in $\mathbf{R}^{n+1}$. Then:

1. $\operatorname{ind}(M) \geq n+2$, with equality if and only if $M$ is a Clifford hypersurface.

2. $\lambda_{2}-\lambda_{1} \leq n-1$, with equality if and only if $\|A\|^{2} \equiv\left|\lambda_{2}\right|$.

The symmetry assumption here seems weak because it holds even when $M$ has only antipodal symmetry, and all of the many known embedded minimal hypersurfaces of spheres do have antipodal symmetry. Indeed, it seems possible that every compact, oriented minimal hypersurface in $S^{n}$ must have this symmetry or, at least, that a minimal hypersurface in $S^{n}$ without this symmetry must have high index.

We therefore have the following corollary:

Corollary 1.4. Let $M$ be a compact, oriented minimal non-equatorial hypersurface of $S^{n}$ invariant under the antipodal map. Then $\operatorname{ind}(M) \geq n+2$, with equality if and only if $M$ is a Clifford hypersurface.

Before I proceed, I would like to thank my advisor, Professor Bruce Solomon, for his lessons on mathematics and for his supervision that guided me to the understanding and achievement of this paper which is part of my Ph.D. dissertation [7].

2. Some Preliminaries. For every fixed vector $w$ in $\mathbf{R}^{n+1}$ we define $f_{w}(m)=$ $\langle w, \nu(m)\rangle$ and $l_{w}(m)=\langle w, m\rangle$ for all $m \in M$. Here $\langle$,$\rangle denotes the inner product$ in $\mathbf{R}^{n+1}$ and $\nu$ denotes a unit normal vector field on $M$. As explained in $[10, \S 1]$ the minimality of $M$ implies

$$
-\Delta l_{w}=(n-1) l_{w}
$$

and the Codazzi equations imply

$$
-\Delta f_{w}=\|A\|^{2} f_{w}
$$

This latter equation makes the functions $f_{w}$ eigenfunctions of $J$ with eigenvalue $-(n-1)$.

Throughout this paper we will denote by $\lambda_{1}=\lambda_{1}(M)$ the first eigenvalue of the Jacobi operator $J$. It is well-known that the multiplicity of $\lambda_{1}$ is 1 and that the eigenfunctions associated to $\lambda_{1}$ never vanish on $M$, (e.g. by Courant's nodal domain theorem, [2]).

In proving our Main Theorem we will use the following two results:

Theorem 2.3. ([9, Lemma 6.1.7]) If $M$ is not totally geodesic, then $\lambda_{1} \leq$ $-2(n-1)$. 
Theorem 2.4. ([1] and [6]) The Clifford hypersurfaces are the only minimal hypersurfaces of $S^{n}$ with $\|A\|^{2} \equiv n-1$.

We will need the following result by Do Carmo, Ritore and Ros in section 4.

TheOREM 2.5. ([3, Theorem 3]) If $M$ has antipodal symmetry and its unit normal vector field is odd, then there exists an even eigenfunction $\psi$ of the Jacobi operator, with $J(\psi)=\lambda \psi$ and $\lambda \in\left(\lambda_{1}, 0\right)$.

3. Minimal Hypersurfaces with Low Index and Weak Symmetry. In this section we prove Theorems 1.2 as stated above. We begin with three lemmas:

LEMMA 3.1. If $M$ is a compact, orientable non-equatorial minimal hypersurface of $S^{n}$, then $\operatorname{ind}(M) \geq n+2$. Moreover $-(n-1)$ is an eigenvalue of $J$ with multiplicity greater than or equal to $n+1$

Proof. Let us define the following linear subspace of $C^{\infty}(M)$

$$
\Gamma=\left\{f_{v}: M \longrightarrow \mathbf{R}, \quad f_{v}(m)=\langle v, \nu(m)\rangle \forall m \in M, \quad v \in \mathbf{R}^{\mathbf{n}+\mathbf{1}}\right\}
$$

Our goal is to prove $\operatorname{dim}(\Gamma)=n+1$. For in this case, Equation 2.2 above shows that the multiplicity of the eigenvalue $-(n-1)$ is a least $n+1$. It then follows that $\operatorname{ind}(M) \geq n+2$, because besides the eigenvalue $-(n-1)$, we have the negative eigenvalue $\lambda_{1} \leq-2(n-1)$ by Simons' theorem above. We proceed by showing that when $\operatorname{dim}(\Gamma)<n+1, M$ is totally geodesic. Suppose $\operatorname{dim}(\Gamma)<n+1$. Then there exists a unit vector $v \in \mathbf{R}^{n+1}$, such that $f_{v} \equiv 0$ on $M$, i.e. $\langle\nu(m), v\rangle=0$ for all $m \in M$. For each $x \in S^{n}$, let $v^{T}(x)$ be the tangential projection of $v$ onto $T_{x} S^{n}$. Since $\langle\nu(m), v\rangle=0, v^{T}$ defines a vector field tangent to $M$ itself. So for any $m \neq \pm v$ in $M$, the integral curve of $v^{T}$ passing through $m$ is a great semicircle containing $v$ and $m$, and is contained in $M$. This forces $M$ to equal the totally geodesic $S^{n-1}$ containing $v$ and tangent to $T_{v} M$.

Corollary 3.2. If ind $(M)=n+2$ then $\lambda_{2}=-(n-1)$.

Proof. Lemma 3.1 makes clear that when $\operatorname{ind}(M)=n+2$, we have that the only negative eigenvalues of $J$ are $\lambda_{1}$, with multiplicity 1 , and $-(n-1)$, with multiplicity $n+1$.

Lemma 3.3. Let $M$ be a minimal hypersurface in $S^{n}$. If $\rho$ is a non-vanishing eigenfunction corresponding to $\lambda_{1}$, and if $f$ is any function perpendicular to $\rho$ in $L^{2}(M)$, i.e. $\int_{M} \rho f=0$, then:

$$
\int_{M} J(f) f \geq \lambda_{2} \int_{M} f^{2} \text { and equality holds iff } \quad J(f)=\lambda_{2} f
$$

Proof. The Lemma follows from the minimax (i.e Rayleigh quotient) characterization of eigenvalues for self-adjoint elliptic operators.

Lemma 3.4. Under the hypotheses of Theorem 1.3 we have $\int_{M} l_{w} \rho^{2}=0$ for every $w \in \mathbf{R}^{n+1}$

Proof. First, notice that since $J$ is invariant under isometries of $M$ and the multiplicity of $\lambda_{1}$ is $1, \rho$ must be invariant under isometries in $O_{M}(n+1)$. Since $M$ is a compact manifold, $O_{M}(n+1)$ is a closed subgroup of $O(n+1)$, hence a compact Lie group. Let $d \mu$ be the bi-invariant measure on $O_{M}(n+1)$ such that $\int_{O_{M}(n+1)} d \mu=1$ (if $O_{M}(n+1)$ is finite, this measure simply places a point mass of equal size at each element of $O_{M}(n+1)$, and the integral is just a finite sum). Then we have: 


$$
\begin{aligned}
\int_{m \in M} l_{w} \rho^{2} & =\int_{m \in M} \rho^{2}(m)\langle m, w\rangle \\
& =\int_{g \in O_{M}(n+1)}\left(\int_{g^{-1} M} \rho^{2}(m)\langle w, m\rangle\right) d \mu(g) \\
& =\int_{g \in O_{M}(n+1)}\left(\int_{m \in M} \rho^{2}(g m)\langle w, g m\rangle\right) d \mu(g) \\
& =\int_{g \in O_{M}(n+1)}\left(\int_{m \in M} \rho^{2}(m)\left\langle g^{-1} w, m\right\rangle\right) d \mu(g) \\
& =\int_{m \in M} \rho^{2}(m)\left\langle\int_{g \in O_{M}(n+1)} g^{-1} w d \mu(g), m\right\rangle \\
& =0
\end{aligned}
$$

since the vector $\int_{O_{M}(n+1)} g^{-1} w d \mu(g)$ is clearly fixed by $O_{M}(n+1)$ and therefore vanishes, by assumption.

We are now ready to give our main argument. For the reader convenience we will restate Theorem 1.3.

THEOREM 1.3. Let $M$ be a compact, oriented minimal non-equatorial hypersurface of $S^{n}$, and suppose that $O_{M}(n+1)$ fixes only the origin in $\mathbf{R}^{n+1}$. Then:

1. $\operatorname{ind}(M) \geq n+2$, with equality if and only if $M$ is a Clifford hypersurface.

2. $\lambda_{2}-\lambda_{1} \leq n-1$, with equality only if $\|A\|^{2} \equiv\left|\lambda_{2}\right|$.

Proof. We will prove part 2 first. Choose an arbitrary $w \in \mathbf{R}^{n+1}$. Since $-\Delta \rho-\|A\|^{2} \rho=\left(\lambda_{1}+n-1\right) \rho$ and $-\Delta l_{w}=(n-1) l_{w}$ we have:

$$
\begin{aligned}
\Delta\left(\rho l_{w}\right) & =\rho \Delta l_{w}+l_{w} \Delta \rho+2\left\langle\nabla \rho, \nabla l_{w}\right\rangle \\
& =\left(-\lambda_{1}-2(n-1)\right) \rho l_{w}-\|A\|^{2} \rho l_{w}+2\left\langle\nabla \rho, \nabla l_{w}\right\rangle
\end{aligned}
$$

Therefore

$$
\begin{aligned}
\int_{M} J\left(\rho l_{w}\right) \rho l_{w} & =\left(\lambda_{1}+(n-1)\right) \int_{M}\left(\rho l_{w}\right)^{2}-2 \int_{M} \rho l_{w}\left\langle\nabla \rho, \nabla l_{w}\right\rangle \\
& =\left(\lambda_{1}+(n-1)\right) \int_{M}\left(\rho l_{w}\right)^{2}-\int_{M} \rho\left\langle\nabla \rho, \nabla l_{w}^{2}\right\rangle \\
& \geq \lambda_{2} \int_{M}\left(\rho l_{w}\right)^{2}
\end{aligned}
$$

In the last inequality we have used Lemma 3.3 and Lemma 3.4. From the estimate above we deduce:

$$
\int_{M} \rho\left\langle\nabla \rho, \nabla l_{w}^{2}\right\rangle \leq\left(\lambda_{1}-\lambda_{2}+(n-1)\right) \int_{M}\left(\rho l_{w}\right)^{2}
$$

We know that $\sum_{i=1}^{n+1}\left(l_{e_{i}}\right)^{2} \equiv 1$ for any orthonormal basis $\left\{e_{i}\right\}_{i=1}^{n+1}$ of $\mathbf{R}^{n+1}$, therefore if we apply the inequality above to $l_{w}=l_{e_{i}}, i=1, \ldots, n+1$ and add these inequalities we get

$$
\left(\lambda_{1}-\lambda_{2}+(n-1)\right) \int_{M} \rho^{2} \geq 0
$$


Hence the distance between the first and second eigenvalue is a most $n-1$. We also have that if $\lambda_{2}-\lambda_{1}=n-1$ then the right hand side of (3.7) vanishes and therefore the symmetric bilinear form

$$
\xi: \mathbf{R}^{n+1} \times \mathbf{R}^{n+1} \longrightarrow \mathbf{R} \quad \text { given by } \quad \xi(w, v)=\int_{M} \rho\left\langle\nabla \rho, \nabla\left(l_{w} l_{v}\right)\right\rangle
$$

satisfies $\xi(w, w) \leq 0$ for any $w \in \mathbf{R}^{n+1}$. Using again that $\sum_{i=1}^{n+1}\left(l_{e_{i}}\right)^{2} \equiv 1$ for any orthonormal basis $\left\{e_{i}\right\}_{i=1}^{n+1}$ of $\mathbf{R}^{n+1}$, we conclude that $\xi$ is traceless. Consequently $\xi(w, w)=0$ for all $w \in R^{n+1}$. Since $\lambda_{2}-\lambda_{1}=n-1$ and $\xi(w, w)=0$, (3.6) reduces to:

$$
\int_{M} J\left(\rho l_{w}\right) \rho l_{w}=\lambda_{2} \int_{M}\left(\rho l_{w}\right)^{2}
$$

This equality together with Lemma 3.3 tell us that:

$$
J\left(\rho l_{w}\right)=\lambda_{2} \rho l_{w}
$$

Using equation (3.5) to rewrite the equality above gives us $2\left\langle\nabla \rho, \nabla l_{w}\right\rangle=2\langle\nabla \rho, w\rangle=0$. Since $\nabla l_{w}$ is the tangential component of $w$ in $T_{m} M$, and $w$ was arbitrary, $\nabla \rho$ must vanish. Hence $\rho$ is constant. Notice that $\rho$ constant implies $\|A\|$ constant. Finally $\lambda_{2}-\lambda_{1}=n-1$ forces $\|A\|^{2}$ to be equal to $-\lambda_{2}$ and Part 1 follows. Let us prove Part 1. By Corollary 3.2, ind $(M)=n+2$ implies that $\lambda_{2}=-(n-1)$. Since $M$ is not totally geodesic we have that $\lambda_{1} \leq-2(n-1)$, Simons' result (Theorem 2.3), therefore the distance between $\lambda_{1}$ and $\lambda_{2}$ is greater than or equal to $n-1$. By Part 1 , we have that this distance is exactly $n-1$ and again by Part 1, this implies that $\|A\|^{2} \equiv(n-1)$. Theorem (2.4) (The uniqueness theorem of Chern-DoCarmo-Kobayashi and Lawson), now implies the result.

We have been trying, so far without success, to remove the symmetry condition in the result above. Even though all known examples of embedded hypersurfaces of $S^{n}$ have antipodal symmetry, it seems plausible that one might be able to construct a minimal hypersurface of $S^{n}$ without this symmetry using, e.g., the gluing technique of Kapouleas and Yang [5]. This antipodal condition is not new; it appears naturally when working with integral geometry. For example, Ros [8] and independently Topping [11], proved that the Willmore conjecture holds for surfaces invariant under the antipodal map, i.e., they proved that the square of the mean curvature of a torus immersed in $\mathbf{R}^{3}$ must always take a value no less than $2 \pi^{2}$ if the image of this torus under the stereographic projection in $S^{3}$ has antipodal symmetry.

REMARK 3.8. The idea of picking the test functions $\rho l_{w}$ relies on the following identity:

$$
\int_{M} J(f g) f g=\int_{M} J(f) f g^{2}+\int_{M} f^{2}|\nabla g|^{2}
$$

In order to show the existence of an eigenvalue of $J$ between $\lambda_{1}$ and $-(n-1)$ or to get a contradiction otherwise, we must take a test function, $f g$, that makes the expression on the left of (3.9) as negative as possible while $\int_{M} \rho f g=0$. Notice that $f=\rho$ makes the first term on the right of (3.9) as negative as $\lambda_{1} \int_{M}(f g)^{2}$. Since the second term on the right of (3.9) contains an expression of the form $|\nabla g|^{2}$ we picked 
for $g$ an eigenfunction of $-\Delta$ corresponding to a small eigenvalue. Namely, we took $g=l_{w}$.

REMARK 3.9. By Equation (2.1) we have that the coordinate functions are eigenfunctions of $-\Delta$ with eigenvalue $n-1$. Yau's conjecture states that if $M$ is not only immersed but embedded, then $n-1$ is the first eigenvalue of $-\Delta$. Notice that one way to prove that Yau's conjecture is not true is to show the existance of a non-zero function $f$ with

$$
\int_{M} f=0 \text { and } \int_{M}|\nabla f|^{2}<(n-1) \int_{M} f^{2}
$$

Using Part 2 of Theorem 1.3 and the identity (3.9) we have that for any minimal embedded hypersurface in $S^{n}$ with antipodal symmetry and $\|A\|^{2}$ non constant, the function $g$ with $g \rho$ an eigenfunction of $J$ corresponding to $\lambda_{2}$ satisfies that:

$$
\int_{M} \rho^{2} g=0 \text { and } \int_{M} \rho^{2}|\nabla g|^{2}=\left(\lambda_{2}-\lambda_{1}\right) \int_{M} \rho^{2} g^{2}<(n-1) \int_{M} \rho^{2} g^{2}
$$

4. Stability Index Jump for Non-totally Geodesic Minimal Hypersurfaces in $S^{n}$ that are not Clifford Hypersurfaces. In this section we will give partial solution to conjecture 1.2 in $\S 1$. Conjecture 1.2 is motivated by the behavior of the index functional on the space of minimal hypersurfaces in $S^{n}$ with $\|A\|^{2}$ constant. For these hypersurfaces we have, in general, that the space $\left\{a+f_{w}+l_{v}: a \in \mathbf{R}^{1} w, v \in\right.$ $\left.\mathbf{R}^{n+1}\right\}$ gives a $2 n+3$ dimensional space in which the index form is negative definited. The exceptions are the equators and the Clifford hypersurfaces. For the equators due to the their big symmetry group, the space $\left\{f_{w}: w \in \mathbf{R}^{n+1}\right\}$ collapses to the space of constant functions, and because equators are totally geodesic, $\|A\|^{2} \equiv 0$, then the index form is not negative definite on the space $\left\{l_{w}: w \in \mathbf{R}^{n+1}\right\}$ but identically zero. Hence the index of the equators is just 1. For Clifford hypersurfaces, due to their symmetries, the spaces $\left\{f_{w}: w \in \mathbf{R}^{n+1}\right\}$ and $\left\{l_{w}: w \in \mathbf{R}^{n+1}\right\}$ are the same. Hence the index of the Clifford hypersurfaces is just $n+2$.

LEMMA 4.1. Let $M \subset S^{n}$ be a non-equatorial minimal hypersurface with antipodal symmetry that is not Clifford. If $\rho, f_{w}$ and $l_{w}$ are defined as in sections 2 and 3, then the dimension of the space $\Upsilon=\left\{a \rho+f_{w}+l_{v}: a \in \mathbf{R}^{1} w, v \in \mathbf{R}^{n+1}\right\}$ is $2 n+3$.

Proof: We proceed by showing that when $\operatorname{dim}(\Upsilon)<2 n+3, M$ is either totally geodesic or a Clifford hypersurface. Suppose $\operatorname{dim}(\Upsilon)<2 n+3$. Then, there exist $a \in \mathbf{R}^{1}, w, v \in \mathbf{R}^{n+1}$ not all of them zero, such that $a \rho+f_{w}+l_{v} \equiv 0$. If $a=0$, $w=0$ and $v \neq 0$ then it is clear that $M$ is the equator $\left\{x \in S^{n}:\langle x, v\rangle=0\right\}$. If $a=0$, $v=0$ and $w \neq 0$ then it follows from Lemma 3.1 that $M$ is an equator. If $a=0$, $w \neq 0$ and $v \neq 0$ it follows from equation 2.1 and 2.2 that $\|A\|^{2} \equiv n-1$, therefore by Theorem 2.4, $M$ is Clifford. The case $a \neq 0$ is impossible because it would imply that $\rho=f_{w}+l_{v}$ for some $w, v \in \mathbf{R}^{n+1}$. Since $\rho$ is an even function and $l_{v}$ is an odd function, we get that for any $m \in M, 2 l_{v}(m)=f_{w}(m)-f_{w}(-m)$. Then either $2 l_{v} \equiv 0$ or $2 l_{v}(m)=-2 f_{w}(m)$; both cases lead to a contradiction with the equation $\rho=f_{w}+l_{v}$.

Proposition 4.2. Let $M$ be a hypersurface like in the previous lemma. If the unit normal vector is even, then the ind $(M) \geq 2 n+3$. 
Proof. By Lemma 4.1 and the definition of index, it is enough to show that the index form is negative definite on $\Upsilon$. Since $\rho$ and $f_{w}$ are eigenfunctions of $J$ with different eigenvalues, we get

$$
\int_{M} \rho f_{v}=0
$$

Since the functions $f_{w}$ are even by assumption and the functions $l_{v}$ are odd, then the functions $f_{w} l_{v}$ and $\rho l_{v}$ are odd, therefore

$$
\int_{M} f_{w} l_{v}=0=\int_{M} \rho l_{v}
$$

By Green's theorem, (2.1) and (2.2) we get that

$$
\int_{M}\|A\|^{2} f_{w} l_{v}=(n-1) \int_{M} f_{w} l_{v}=0
$$

Using (4.2.1)-(4.2.3) we have for any non-zero function $f=a \rho+f_{w}+l_{v} \in \Upsilon$ that:

$$
\begin{aligned}
I(f, f) & =\int_{M} J(f) f=\int_{M}\left(\lambda_{1} a \rho-\|A\|^{2} l_{v}-(n-1) f_{w}\right) f \\
& =\int_{M}\left\{\lambda_{1} a^{2} \rho^{2}-(n-1) f_{w}^{2}-\|A\|^{2} l_{w}^{2}\right\}<0
\end{aligned}
$$

Therefore the index form is negative defined in $\Upsilon$.

Notice that Proposition 4.2 along with Theorem 2.5 gives a different proof of Corollary 1.4.

REMARK. We can deduce the same conclusion in Proposition 4.2 by changing the hypothesis "unit normal vector field even" by the hypothesis " $\|A\|^{2} \geq n-1$ ". Once again the proof consists in verifying that the index form is negative definite on $\Upsilon$.

Theorem 4.3. Let $M$ be a minimal non-equatorial hypersurface in $S^{n}$ invariant under the antipodal map. If $M$ is not a Clifford hypersurface then $\operatorname{ind}(M) \geq n+4$.

Proof. By Proposition 4.2, we only have to take care of the case when the unit normal vector $\nu$ of $M$ is odd. Suppose $\nu$ is odd. By Theorem 2.5 there exists at least one even eigenfunction $\psi$ of $J$ with $J(\psi)=\lambda \psi$, and $\lambda \in\left(\lambda_{1}, 0\right)$. Therefore we have the following $n+3$ linear independent index eigenfunctions (eigenfunctions of $J$ associated with negative eigenvalues) $\rho, \psi$ and $\left\{f_{e_{i}}\right\}_{i=1}^{n+1}$ where $\left\{e_{i}\right\}_{i=1}^{n+1}$ is any orthonormal basis of $\mathbf{R}^{n+1}$. The argument that guarantee another index eigenfunction is similar to the argument in the proof of the main theorem. Let us assume that the index is $n+3$ (in particular we are assuming that the only negative eigenvalues of $J$ are $\lambda_{1},-(n-1)$ and $\lambda)$. Since $\psi$ is an even function, then $\int_{M} \psi \rho l_{w}$. This allows us to deduce the inequality (3.6) with $\lambda_{2}=-(n-1)$, namely:

$$
\begin{aligned}
\int_{M} J\left(\rho l_{w}\right) \rho l_{w} & =\left(\lambda_{1}+(n-1)\right) \int_{M}\left(\rho l_{w}\right)^{2}-\int_{M} \rho\left\langle\nabla \rho, \nabla l_{w}^{2}\right\rangle \\
& \geq-(n-1) \int_{M}\left(\rho l_{w}\right)^{2}
\end{aligned}
$$


By Theorem 2.3 and the minimax characterization of eigenvalues, the inequality above can only be fulfilled if $\lambda_{1}=-2(n-1)$ and $\int_{M} \rho\left\langle\nabla \rho, \nabla l_{w}^{2}\right\rangle=0$ for all $w \in \mathbf{R}^{n+1}$. Using again the minimax characterization we deduce that $J\left(\rho l_{w}\right)=-(n-1) l_{w}$, which along with $\lambda_{1}=-2(n-1)$ imply that $\|A\|^{2}=n-1$. This contradicts Theorem 2.4 since we are assuming that $M$ is not Clifford.

\section{REFERENCES}

[1] S.S. Chern, M. DoCarmo, ANd S. Kobayashi, Minimal submanifolds of a sphere with second fundamental form of constant length, Functional Analysis and Related Fields, Proc. Conf. M. Stone, Springer, 1970, pp. 59-75.

[2] Chavel IsaAc, Eigenvalues in Riemannian Geometric, Pure and Applied Mathematics., 115 Academic Press.

[3] Do-Carmo, M., Ritore, M., \& Ros, A., Compact minimal hypersurfaces with index one in the real projective space, Comment. Math. Helv., 75 (2000), pp. 247-254.

[4] T. Frankel, On the fundamental group of a compact minimal submanifold, Ann. of Math., 83 (1966), pp. 68-73.

[5] N. KAPOULEAS, S-D. YANG, A doubling construction for minimal surfaces, in preparation.

[6] Lawson, H. B, Local rigidity theorems for minimal hypersurfaces, Ann. of Math. (2), 89 (1969), pp. 187-197.

[7] O. Perdomo, First eigenvalue and index: Two characterizations of minimal Clifford hypersurfaces of spheres, Ph.D. Thesis, Indiana University, 2000.

[8] A. Ros, The Willmore conjecture in the real projective space, Math. Res. Lett., 6 (1999), pp. 487-493.

[9] J. Simons, Minimal Varieties in Riemannian manifolds, Ann. of Math., 88 (1968), pp. 62-105.

[10] B. Solomon, Quartic isoparametric hypersurfaces and quadratic forms, Math. Ann, 293 (1992), pp. 387-398.

[11] P. ToPPING, Towards the Willmore conjecture, Calculus of Variations and PDE (to appear).

[12] F. Urbano, Minimal surfaces with low index in the three dimensional sphere, Proceedings of the American Mathematical Society, v. 108, 4, April 1990, pp. 989-992. 
